\title{
Meeting of The American Pediatric Society
}

\author{
Atlantic City, New Jersey, April 30, 1970
}

Plenary Sessions ${ }^{1}$

Role of Nasopharyngeal Tonsils in the Mechanism of Mucosal Immunity to Poliovirus. Pearay L. Ogra, Dept. of Ped., State Univ., of New York at Buffalo (introduced by David T. Karzon).

Richard W.Newcomb (Children's Asthma Research Institute, Denver, Colo.): Do you have any idea how the antibody titers in your nasal fluids relate to immunoglobulin concentrations?

Dr. OGRA: We are working on it at the moment. It seems that total gamma-A-immunoglobulin concentration decreases by about twofold, but it is not as constant as the drop in the specific viral antibody.

E. RICHARD STIEHM (UCLA Center for the Health Sciences): What is the relative contribution of the tonsillectomy and the adenoidectomy to the immune deficiency noted?

Dr. OGRa: It is very hard these days to pick up children who have had isolated tonsillectomies. I do not have any information on the individual participation of tonsils and adenoids. Most of the children we studied had complete removal of tonsils as well as adenoids.

$A$ Controlled Evaluation of the Effectiveness of Comprehensive Pediatric Care in Influencing Patient Compliance. LeON Gordis and Mil ton Markowitz, Hebrew Univ.-Hadassah Med. Sch., Jerusalem, and Univ. of Conn. Sch. of Med. Hartford Conn.

Alvin H. Novack (New Haven, Gonn.): Dr. GoRDIs, I want to indicate that it is important that we measure the differences between comprehensive and episodic care. I would, however, raise a question as to the definition of comprehensive care. I think you have measured the difference between comprehensive physician care versus episodic physician care. Comprehensive health care must be defined more broadly than just physician care and should include social and psychiatric (mental health) in addition to the usual medical, nursing, and dental health care.

Secondly, it is important to consider the difference between comprehensive and episodic care. The latter is disease oriented, and the former is health promoting, and it is difficult to compare the two when one talks about a chronic disease and compliance.

Dr. GoRDIs: In response to your first question, it seems to me that in analyzing comprehensive care we have to study each of the components that go into iteach component individually as well as all the components together-if we are to find which factors are critical and which factors are extraneous. Although it is

${ }^{1}$ Abstracts appeared in Pediat. Res.4: 371-374 (1970). true that this study focused only on comprehensive care by a physician, the care was not restricted to a single organ system or set of problems. But I am in full agreement with you that different types of studies should also be done, including those of other health professionals, and in different settings.

As far as it being disease oriented, I agree with you there, too. The problem is that the research in this area cannot be done cosmically; we must isolate the specific factors which we wish to analyze, and submit them to rigorous evaluation. I would suggest, therefore, that this type of study should be extended to preventive health services and health care, as well as to chronic disease management.

William ObRINSkx (Montefiore Hospital, NewYork, N.Y.): Comprehensive care is a way of life. It is not something that you can decide on one day to turn on, and a year later to turn off. You have had a population that has never had any experience with comprehensive care, and it takes a lot longer than 1 year to begin to teach the principles of comprehensive care. I would be very much interested in a similar study that might be done with a population who from very early infancy had comprehensive care over a longer period than 1 year.

Joel J. Alpert (Children's Hospital Medical Center, Harvard Medical School, Boston, Mass.) : Dr. GorDIs, I agree with your expressed philosophy very much. We need controlled evaluations of comprehensive care. The challenge, however, is not only to try to measure differences but also to offer explanations as to why differences were or were not found. Thus, other points might be that these were families that were engaged in an identical physical place; that this was care given to individual patients and not to families.

Your report is very similar to some of our own work. We did not measure differences in the first year of our study on 551 families but saw differences in the second and third.

We also found that many of these measured differences disappeared in the third year of the study and these disappearing differences were due to those families on welfare who were pulled back into what we call the welfare-fragmented system by pressures outside of the comprehensive care program. What may, indeed, be needed is a total change in the health care system. Perhaps you have also identified a very complex population. I wonder how many of your 77 patients were on welfare.

Dr. GoRDIs: Only a relatively small proportion were on welfare, but the group was too small to be able to subject it to this type of analysis. 